\title{
Gestational Weight Gain in Japanese Women With Favorable Perinatal Outcomes
}

\author{
Shunji Suzuki
}

\begin{abstract}
Background: We examined the optimal gestational weight gain (GWG) in the healthy Japanese women with favorable perinatal outcomes of singleton pregnancy.

Methods: We calculated the average GWG in the women whose height was $150-164 \mathrm{~cm}$ with favorable perinatal outcomes set for this study. The women were categorized to underweight, normal, overweight and obese based on the pre-pregnancy body mass index categories according to the Institute of Medicine guideline.
\end{abstract}

Results: The average GWG in the normal-weight women with the favorable perinatal outcomes was $11.4 \pm 3.7 \mathrm{~kg}$. It was not significantly different from that in the underweight and overweight women (12.0 \pm 3.4 and $10.0 \pm 4.8 \mathrm{~kg}$ ) by Student's $t$-test. The average GWG in the obese women was significantly lower than that in the other three groups $(3.2 \pm 2.2 \mathrm{~kg}, \mathrm{P}<0.01)$.

Conclusion: Based on the current results, the optimal GWG for the Japanese women without obesity was found to be $10-12 \mathrm{~kg}$.

Keywords: Gestational weight gain; Japanese women; Favorable perinatal outcomes

\section{Introduction}

Gestational weight gain (GWG) has been reported to be associated with the short- and long-term perinatal outcomes of both mother and children [1-4]. Insufficient and excessive GWG seems to be associated with adverse perinatal outcomes. Therefore, examinations concerning optimal GWG are very important. To date, the goals of the previous studies concerning the GWG have seemed to set the recommended ranges of GWG in women of various physiques [2-4]. However, optimal GWG has not yet been clearly defined and remains one of the

Manuscript accepted for publication November 11, 2016

Department of Obstetrics and Gynecology, Japanese Red Cross Katsushika Maternity Hospital, 5-11-12 Tateishi, Katsushika-ku, Tokyo 124-0012, Japan. Email:czg83542@mopera.ne.jp

doi: https://doi.org/10.14740/jocmr2810w most controversial issues in modern perinatal medicine.

Because some birth cohort studies in Japan have also been believed to be suitable for epidemiological studies to demonstrate the "developmental origins of health and disease" indicating the developmental plasticity and the mismatch concept, the importance of nutrition and weight gain during pre-pregnancy and pregnancy has now been widely recognized in Japan [5-7].

Based on our previous observation in Japan [8], the GWG was indicated to be more tolerant than ever $[9,10]$, especially in overweight women. However, we could not indicate the recommended ranges of GWG in Japanese women clearly. In this study, therefore, we calculated the optimal GWG in the pregnant Japanese women with favorable perinatal outcomes.

\section{Methods}

The protocol for this study was approved by the Ethics Committee of the Japanese Red Cross Katsushika Maternity Hospital. Informed consent concerning analysis from a retrospective database was obtained from all subjects.

We reviewed the obstetric records of singleton pregnant Japanese women who delivered singleton neonates at our institute at $\geq 22$ weeks' gestation from April 2012 through September 2016.

In order to examine the optimal GWG in Japanese women, we calculated the average GWG in the women whose height was $150-164 \mathrm{~cm}$ (cf. the average height of Japanese adult female: $158 \mathrm{~cm}$ [11]) with favorable perinatal outcomes set for this study as follows: 1) those without medical histories such as pregnancy-induced hypertension, chronic hypertension, diabetes mellitus, renal disease, idiopathic thrombocytopenia and other systemic illnesses, 2) no smoking, 3) normal delivery at 39 - 40 weeks' gestation, 4) postpartum hemorrhage $<800 \mathrm{~mL}, 5$ ) no maternal complications requiring treatment within 1 month after delivery, 6) neonatal birth weight: 3,000 - 3,499 g, 7) neonatal Apgar score at $1 \mathrm{~min} \geq 8$, and 8) no neonatal complications requiring treatment within 1 month after birth. These women were categorized to the underweight (pre-pregnancy body mass index $(\mathrm{BMI})<18.5 \mathrm{~kg} / \mathrm{m}^{2}$ ), normal (pre-pregnancy BMI: 18.5 - $24.9 \mathrm{~kg} / \mathrm{m}^{2}$ ), overweight (pre-pregnancy BMI: 25.0 - $29.9 \mathrm{~kg} /$ $\mathrm{m}^{2}$ ) and obese (pre-pregnancy BMI $\geq 30 \mathrm{~kg} / \mathrm{m}^{2}$ ) groups according to the Institute of Medicine (IOM) guideline [1].

Data were expressed as mean \pm standard deviation (SD) or number (percentages). The one-way ANOVA followed by post $h o c$ analysis was used for continuous variables, and the $\mathrm{X}^{2}$ or 
Table 1. Gestational Weight Gain in the Underweight, Normal-Weight, Overweight and Obese Japanese Women With the Favorable Perinatal Outcomes* Whose Height Was $150-164 \mathrm{~cm}$

\begin{tabular}{lllll}
\hline & Underweight & Normal & Overweight & Obese \\
\hline Numbers of women whose height was $150-164 \mathrm{~cm}$ & 458 & 3,852 & 480 & 92 \\
Numbers with favorable perinatal outcomes* & $125(27.3 \%)^{* *}$ & $793(20.6 \%)$ & $90(18.8 \%)$ & $17(18.9 \%)$ \\
Maternal age (years) & $32.4 \pm 5.0$ & $32.5 \pm 5.6$ & $31.4 \pm 5.8$ & $32.4 \pm 5.1$ \\
Nulliparity & $63(50 \%)$ & $357(45 \%)$ & $40(44 \%)$ & $7(41 \%)$ \\
Maternal height $(\mathrm{cm})$ & $158.4 \pm 3.4$ & $158 \pm 4.0$ & $157.9 \pm 4.5$ & $158.2 \pm 3.3$ \\
Maternal weight at pre-pregnancy $(\mathrm{kg})$ & $43.9 \pm 2.9 * *$ & $52.1 \pm 4.6$ & $66.3 \pm 4.7 * *$ & $80.8 \pm 5.0^{* *}$ \\
Maternal weight at delivery $(\mathrm{kg})$ & $55.9 \pm 4.6^{* *}$ & $63.5 \pm 6.0$ & $76.3 \pm 7.3 * *$ & $84.0 \pm 6.2^{* *}$ \\
Weight gain during pregnancy $(\mathrm{kg})$ & $12.0 \pm 3.4$ & $11.4 \pm 3.7$ & $10.0 \pm 4.8$ & $3.2 \pm 2.2^{* *}$ \\
\hline
\end{tabular}

Data are presented as mean \pm standard deviation or number (percentages). *Favorable perinatal outcomes set for this study: 1) those without medical histories such as pregnancy-induced hypertension, chronic hypertension, diabetes mellitus, renal disease, idiopathic thrombocytopenia and other systemic illnesses, 2) no smoking, 3) normal delivery at 39 - 40 weeks' gestation, 4) postpartum hemorrhage < $800 \mathrm{~mL}$, 5) no maternal complications requiring treatment within 1 month after delivery, 6) neonatal birth weight: 3,000 - 3,499 g, 7) neonatal Apgar score at $1 \mathrm{~min} \geq 8$, and 8 ) no neonatal complications requiring treatment within 1 month after birth. ${ }^{*} \mathrm{P}<0.05$ vs. normal group.

Fisher's exact test for categorical variables. Differences with $\mathrm{P}$ $<0.05$ were considered significant.

\section{Results}

There were 6,022 women who delivered singleton neonates at $\geq 22$ weeks' gestation at our institute during the study period. The height of 4,882 women in these $(81.1 \%)$ was $150-164$ $\mathrm{cm}$. Of these, $458(9.4 \%), 3,852(78.9 \%), 480$ (9.8\%) and 92 (1.9\%) women were defined as underweight, normal (-weight), overweight and obese based on the BMI during pre-pregnancy, respectively. Of these, $1,025(21.0 \%)$ women resulted in the favorable perinatal outcomes.

Table 1 shows the GWG in the underweight, normalweight, overweight and obese women with the favorable perinatal outcomes. The rate of women with the favorable perinatal outcomes in the underweight group was significantly higher than that in the other groups as shown in Table $1(\mathrm{P}<0.01)$. The average GWG in the normal-weight women with the favorable perinatal outcomes was $11.4 \mathrm{~kg}$. It was not significantly different from that in the underweight and overweight women. The average GWG in the obese women with the favorable perinatal outcomes was significantly lower than that in the other three groups $(\mathrm{P}<0.01)$.

\section{Discussion}

In this study, the rate of favorable perinatal outcomes in the underweight women seemed to be higher than that of the women with other physique. In some recent studies, the lower prepregnancy BMI has been observed to contribute to the lower incidences of pregnancy-induced hypertension, gestational diabetes mellitus, macrosomia, cesarean delivery, postpartum hemorrhage and post-term delivery although an increasing severity of maternal underweight BMI had been reported to be associated with an increasing risk of premature delivery $[12,13]$.
The current result may be supported by these previous reports $[12,13]$. However, there may be a serious limitation in this regard. Because, the rate of underweight women managed at our institute was $9.4 \%$, although $22.5 \%$ and $16.8 \%$ of Japanese women in their twenties and thirties has been reported to be underweight due mainly to a strong desire to be thin [14]. Therefore, the nutritional environment in the area with our institute located in the poor areas of Tokyo might be particular in Japan.

In the Japanese guidelines [9, 10], for example, the recommended range of GWG for normal-weight women has been $7-12 \mathrm{~kg}$. In this study, the average GWG in the women without obesity associated with the favorable perinatal outcomes seemed to be $11-12 \mathrm{~kg}$; the value seemed to be the upper limit level of recommended range of GWG in the Japanese guidelines $[9,10]$. The current results may also support our findings in a previous study [8]. Therefore, the Japanese obstetricians should be more tolerant for the GWG in Japanese woman than ever. Otherwise, the increased level of the recommended range of GWG should be examined in the Japanese guidelines.

At last, the optimal GWG found in this study seems to be smaller than the recently reported standards generated in the healthy and well-nourished women $(13.7 \mathrm{~kg})$ from different continents, i.e., Asia, Europe, North America, South America and Africa [2]. The average physique of Japanese women is certainly short and slender in comparison to that of the women in other countries; however, a larger prospective study will be required to assess the optimal range of GWG for Japanese women.

\section{Conclusion}

The Japanese obstetricians should be more tolerant for the GWG in Japanese woman than ever. A larger study may be needed.

\section{Disclosures}

The authors report no conflicts of interest. The author is re- 
sponsible for the content and writing of the paper. This study was supported by the Japan Health, Labour and Welfare scientific research subsidies: "Study on the support of nutritional management of pregnant women, mothers and infants".

\section{References}

1. Rasmussen KM, Yaktine AL, Eds. Weight gain during pregnancy: reexamining the guidelines. Washington, DC: National Academies Press; 2009.

2. Cheikh Ismail L, Bishop DC, Pang R, Ohuma EO, Kac G, Abrams B, Rasmussen K, et al. Gestational weight gain standards based on women enrolled in the Fetal Growth Longitudinal Study of the INTERGROWTH-21st Project: a prospective longitudinal cohort study. BMJ. 2016;352:i555.

3. Drehmer M, Duncan BB, Kac G, Schmidt MI. Association of second and third trimester weight gain in pregnancy with maternal and fetal outcomes. PLoS One. 2013;8(1):e54704.

4. Thangaratinam S, Rogozinska E, Jolly K, Glinkowski S, Roseboom T, Tomlinson JW, Kunz R, et al. Effects of interventions in pregnancy on maternal weight and obstetric outcomes: meta-analysis of randomised evidence. BMJ. 2012;344:e2088.

5. Sata F. [Developmental Origins of Health and Disease (DOHaD) and Epidemiology]. Nihon Eiseigaku Zasshi. 2016;71(1):41-46.

6. Gluckman PD, Hanson MA, Beedle AS. Early life events and their consequences for later disease: a life history and evolutionary perspective. Am J Hum Biol. 2007;19(1):1-
19.

7. Gillman MW, Barker D, Bier D, Cagampang F, Challis J, Fall C, Godfrey K, et al. Meeting report on the 3rd International Congress on Developmental Origins of Health and Disease (DOHaD). Pediatr Res. 2007;61(5 Pt 1):625629.

8. Suzuki S. Optimal Weight Gain During Pregnancy in Japanese Women. J Clin Med Res. 2016;8(11):787-792.

9. Minakami H, Maeda T, Fujii T, Hamada H, Iitsuka Y, Itakura $\mathrm{A}$, Itoh $\mathrm{H}$, et al. Guidelines for obstetrical practice in Japan: Japan Society of Obstetrics and Gynecology (JSOG) and Japan Association of Obstetricians and Gynecologists (JAOG) 2014 edition. J Obstet Gynaecol Res. 2014;40(6):1469-1499.

10. Promotion Council for Healthy Parents and Children 21 (second edition) (in Japanese). Ministry of Health, Labour and Welfare, 2015. http://rhino3.med.yamanashi. ac.jp/sukoyaka2/english.html (October 23, 2016).

11. Japanese height (in Japanese). http://www.geocities.jp/ resultri/crankcho/height_j.html (October 23, 2016).

12. Enomoto K, Aoki S, Toma R, Fujiwara K, Sakamaki K, Hirahara F. Pregnancy Outcomes Based on Pre-Pregnancy Body Mass Index in Japanese Women. PLoS One. 2016;11(6):e0157081.

13. Girsen AI, Mayo JA, Carmichael SL, Phibbs CS, Shachar BZ, Stevenson DK, Lyell DJ, et al. Women's prepregnancy underweight as a risk factor for preterm birth: a retrospective study. BJOG. 2016;123(12):2001-2007.

14. The Japanese Ministry of Health, Labour and Welfare: National Health and Nutrition Examination Survey (2014 in Japanese). http://www.mhlw.go.jp/stf/houdou/0000106405.html (October 23, 2016). 